\title{
A FRAMEWORK BASED ON MATLAB/SIMULINK FOR THE SIMULATION OF DES USING PETRI NET MODELS
}

\author{
Alcaraz-Mejia, M. ${ }^{*} \&$ Campos-Rodriguez, . $^{* *}$ \\ *Department of Electronic, Systems and Informatics, ITESO University, 45604 Tlaquepaque, México \\ ${ }^{* * *}$ DB Cloud, Oracle Guadalajara Design Centre, 45116 Zapopan, México \\ E-Mail: mildreth@iteso.mx, raul.campos.rodriguez@oracle.com
}

\begin{abstract}
This paper proposes the construction of a framework for the simulation of Petri net models based on Matlab/Simulink. The building blocks of the proposed framework use some basic elements provided by the SimEvents ${ }^{\circledR}$ toolbox. The objective is to provide a flexible and powerful environment that captures the execution conditions of real systems, besides a natural integration to Matlab environment, for the simulation of Petri nets. This framework simplifies the construction of the models by dragging and dropping blocks, and connecting them together. Moreover, a programmatic approach is possible for the construction of complex models, just as any Simulink model. The rich set of tools, algorithms and visualization mechanisms in the Matlab environment are available for the integration with the Petri net models constructed by using the blocks introduced in this work. This integration allows for a wide range of possibilities for the simulation of Discrete Event Systems modelled as Petri nets. Results obtained within this framework are contrasted with those reported in the original sources. The concluding remarks section provides a link for downloading and testing the library.

(Received in February 2019, accepted in July 2019. This paper was with the authors 2 weeks for 1 revision.)
\end{abstract}

Key Words: Petri Net Models, Discrete-Event Systems, Matlab, Simulink, SimEvents, DiscreteEvent Simulation, Hybrid Simulation

\section{INTRODUCTION}

Petri Nets (PN) are a popular formalism for the design, modelling and simulation of DiscreteEvent Systems (DES). This formalism is widely accepted by practitioners and scientists. A PN model is a directed and bipartite graph with two classes of nodes, places and transitions. Places represent conditions, while transitions represent events. The tokens residing in places of a PN represent the state of the system. Along with the graphical representation, a PN has a strong mathematical basis, which eases the analysis of its properties. The construction of computational algorithms for the PN simulation, performance analysis and parameter tuning, is also possible.

The theory and formal analysis of PN have been widely developed. There are many introductory material and advanced textbooks with formal analysis of PN such as reachability, deadlock avoidance, stability and control theory [1-4]. Some extensions to classical PN formalism have been introduced in the literature such as timed PN [5], continuous and hybrid models [6], coloured and high-level PN [7, 8], stochastic models [9, 10], object-oriented PN [11], among others.

Applications such as modelling, control, and performance analysis of manufacturing process [12], analysis and design of distributed systems [13], design and performance analysis of communication protocols [14], business process modelling and simulation [15], logistics systems [16, 17], digital hardware design [18], among others, have been successfully addressed by using the PN formalism.

In addition, companies, the academia, and the community, have developed some tools and software that support a variety of activities such as design, modelling, simulation, and optimisation of DES as well as of hybrid systems, based on the PN formalism. Some PN editors and analysers, which are written in Java, can be used directly on web pages [19-21]. 
Some tools are commercial and some others are free of charge, as well as supported by different operating systems such as Windows, Linux distributions, iOS, or others [22-28]. A wide list of tools and software is available in Petri Net World page. However, only quite few developments take advantage from frameworks like Simulink [29, 30].

This work introduces a Framework based on Matlab/Simulink for the Simulation of DES by means of Petri net models, using the SimEvents ${ }^{\circledR}$ toolbox as a plus. SimEvents ${ }^{\circledR}$ is a discrete-event engine for the modelling and simulation of DES introduced by MathWorks. The engine includes a set of built blocks for its integration with the Simulink environment for pure discrete event-driven simulation, or even for hybrid simulation. Basic blocks such as queues, servers, routers, switches, entity generators and attributes are included in this engine. This SimEvents blocks are used for the construction of basic components of a PN model such as generic PN Places or Transitions. The generic PN place allows for an arbitrary number of input and output transitions, as well as with an arbitrary number of tokens. Similarly, the generic PN transition allows for an arbitrary number of input and output places. A service time is associated to each transition in such a way that a pure event-driven simulation, or even a hybrid simulation, is possible. Moreover, generic transitions can be configured for single or multiple firing semantics at every simulation step. Finally, this paper proposes a block for the generation of tokens or entities at the beginning of the simulation to set up the initial marking.

Furthermore, the rich set of numeric algorithms, data analysis techniques, the visualization tools, the deployment capabilities in the Matlab framework, and the model-based design abilities of Simulink, are available for the modelling and simulation of DES, and hybrid systems, modelled as PN.

The advantages of the framework herein presented are:

a) The integration of blocks for the construction of PN models in the Matlab/Simulink environment by dragging and dropping places and transitions, connecting them together, and defining the initial conditions for the PN places;

b) The ability of including a wide range of criteria for the selection of output transitions from a place, which can be as simple as a traditional round-robin, Binomial, Poisson distribution, or even real statistical data from a physical system;

c) The ability of including a service time for transitions, which could be a positive integer for a pure event-driven simulation, a real number, a real-evaluated Matlab function, a probability distribution, or even real statistical data from an implemented system;

d) The possibility of monitoring the marking of a place, which leads to a wide set of possibilities for a reachability analysis, and the possibility of monitoring the firing of the transitions, which leads to a wide set of possibilities for a linguistic analysis.

e) The possibility of include programmatic functions in order to extract many data from the model or from performance, in order to improve the model or to apply property analysis.

The rest of this paper organizes as follows: Section 2 introduces the notions and terminology of the PN formalism. Section 3 reviews the main aspects of the SimEvents environment and proposes the subsystems that represent a generic place, a generic transition and the initial marking of a PN model. Section 4 provides a set of application examples reported in the literature. In addition, some simulation experiments are performed by using the proposed blocks. Besides, as a validation process of the simulation, the results obtained within this framework are contrasted with those reported in the original sources of the examples. Finally, conclusions and bibliographical references are given.

\section{PN MODELLING AND SIMULATION FRAMEWORK}

A PN is a mathematical tool widely used for the design, the modelling and the simulation of DES. A PN model is a directed graph with two classes of nodes called places and transitions. 
The arcs of the PN allow for connecting places to transitions, and transitions to places, guiding the dynamic of the net.

Fig. 1 depicts a Petri net model for a disk-scheduling algorithm reported in [31]. The number of places in the net is six, while the number of transitions is five. The PN model captures the dynamic behaviour of an algorithm for the dispatching of disk requests in a computer operating system. A request arrives arbitrarily by firing the corresponding transition in the model and may simulate different workload conditions. A place holds the disk request while waits for the availability of a disk drive. A seek operation starts once the disk drive is successfully allocated. After this operation, the transfer of information begins. Once the transfer operation finishes, the system releases the resources, then it becomes ready for attending further disk requests. The model, with three available controllers and four disk drives, is used for simulation purposes in order to illustrate the usability of the framework proposed in this work.

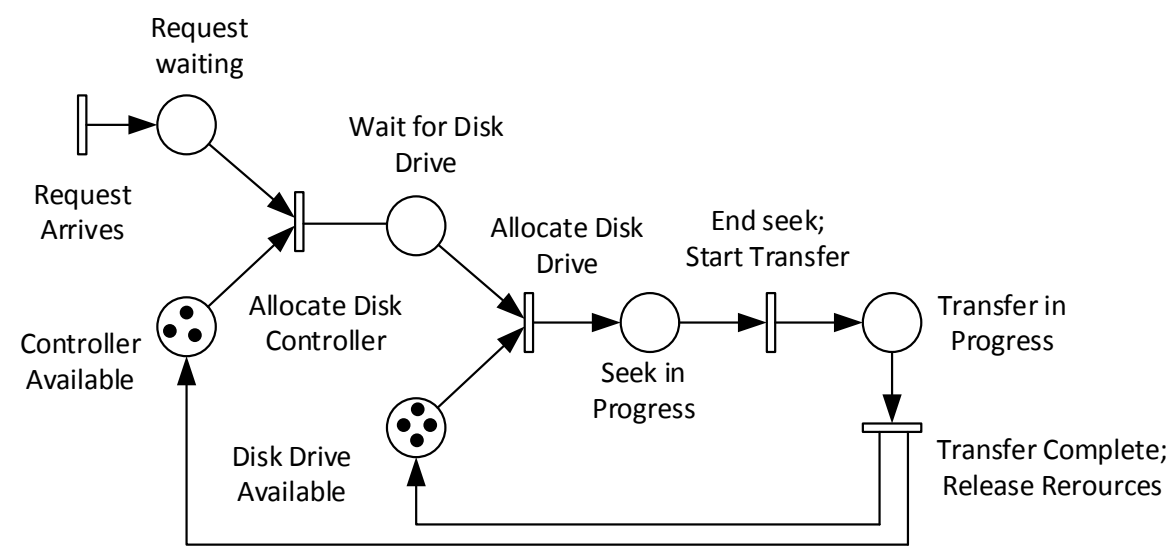

Figure 1: A PN model representing a scheduling algorithm for the allocation of disk controllers and drives.

The herein introduced framework is based on Matlab/Simulink environment, which provides a reach set of tools and algorithms for an easy manipulation of matrices of any type as well as a set of visualization and numerical methods. In particular, SimEvents ${ }^{\circledR}$ offers a wide variety of components for modelling and simulation of DES, such as communication protocols, manufacturing processes, and logistic systems, among others. It includes predefined blocks like servers, queues, switches, routers and entity generators for representing most of the dynamic aspects of a DES. However, for a proper representation of PN models as well as to capture the semantics of the main elements of a PN is necessary to make a suitable arrangement of SimEvents ${ }^{\circledR}$ blocks. The herein proposed PN framework is based on: Server, Queue, Entity Combiner, Replicate, Path Combiner, Output Switch, and Entity Generator, which all are blocks of SimEvents ${ }^{\circledR}$. For a detailed explanation of these blocks, readers are referred to [32].

\section{PETRI NETS SIMULATION FRAMEWORK}

The PN key concepts to include in the herein proposed framework are those of a transition, a place, connection among places and transitions, initial marking, firing activation, marking evolution rule and the concept of a token.

For the construction of these basic elements of a PN model, named blocks as well, is used the capability to create subsystems based on Matlab/Simulink framework. Every block is a subsystem of the Simulink environment with a suitable arrangement of SimEvents blocks such that captures the semantic of the corresponding component of a PN model. Each built block includes a dialog box for adjusting its parameters at design time, e.g. number of inputs 
or outputs for a transition or a place, probability distribution for a place with more than one output, among others.
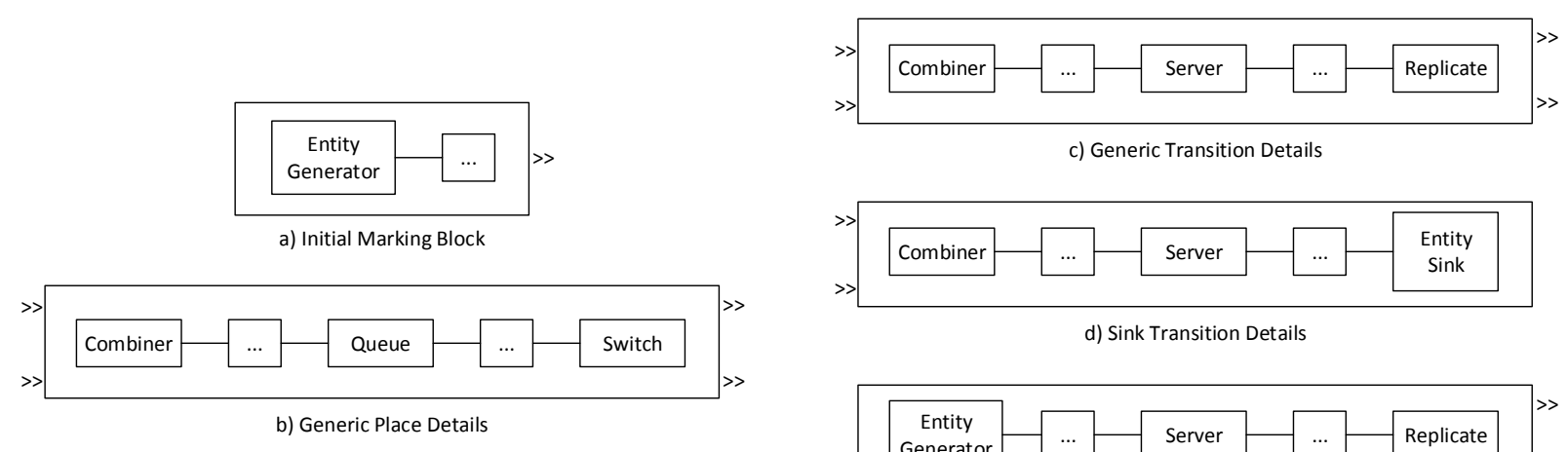

c) Generic Transition Details

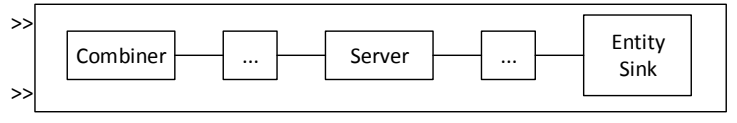

d) Sink Transition Details

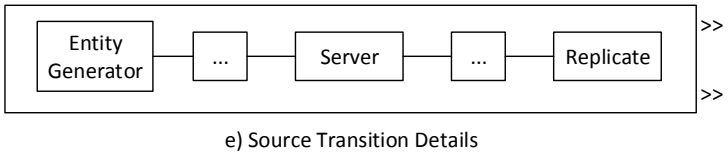

Figure 2: A detailed view of the implementation of basic elements of the PN model.

\subsection{Definition of PN elements as SimEvents blocks}

The blocks proposed to build PN models based on SimEvents are shown in Fig. 2. The description of every block is given in the following.

Initial Marking Block. An entity generator is the basis for the creation of tokens from a PN, as shown in Fig. 2 a. The user configures the number of tokens to produce by means of the block dialog box. After the initialization process, the place connected to this block receives the entities initially generated. Thus, if a place has an initial marking, such a place includes an additional input port solely for its connection with an initial marking block.

Generic Place Block. The core element in the construction of a place is a queue block, as shown in Fig. 2 b. In general, places can hold any number of tokens. However, it is useful to have bounds. Thus, the queue may store a maximum number of entities, called capacity. Since a PN place can receive tokens from any number of input arcs, then a path combiner is coupled to the queue input port. The number of input ports corresponds with the number of input transitions to that place. Symmetrically, a PN place can deliver the tokens through any of its output arcs. Thus, an output switch is connected to the queue output port. The number of output ports corresponds with the number of the output transitions of that place. In this way, the method selected for the switching criterion allows for simulating fairness or competitive conditions in a PN model, such as equiprobable or Bernoulli probability distributions, or even more sophisticated criterion based on complex algorithms implemented in Simulink blocks. This gives a great flexibility for capturing real conditions in the proposed simulation framework.

Generic Transition Block. For the construction of a transition, this work proposes the use of a server block. For the purpose of a pure discrete-event simulation, the service time for the servers, i.e., for each transition in the PN, is fixed to a specific value, such an integer multiple of a fundamental unit of time, e.g., a clock tick. The default service time for the Generic Transition is set up to one time unit. Additionally, a transition fires whenever all its preconditions are satisfied. To meet with this criterion, an entity combiner is added at the input of the subsystem that conforms the transition. This allows for specifying the number of entities that have to be present before server starts serving. Indeed, the entity combiner produces a new compound entity for a given set of incoming entities, whenever all of them are present at its input ports at the same instant. In this way, for example, if a transition has two input places, then the entity combiner have to wait until at least one entity arrives from each of its input ports, preventing that the server starts serving until all of the conditions are satisfied. Similarly, the firing of a transition in a PN produces the number of tokens specified 
by its output set. Therefore, the subsystem couples the output port of the server to a replicate block. In this way, the number of tokens that a transition firing has to produce is established by fixing the number of entities to be copied at the replicate block. The main components of the generic transition block are depicted in Fig. $2 \mathrm{c}$.

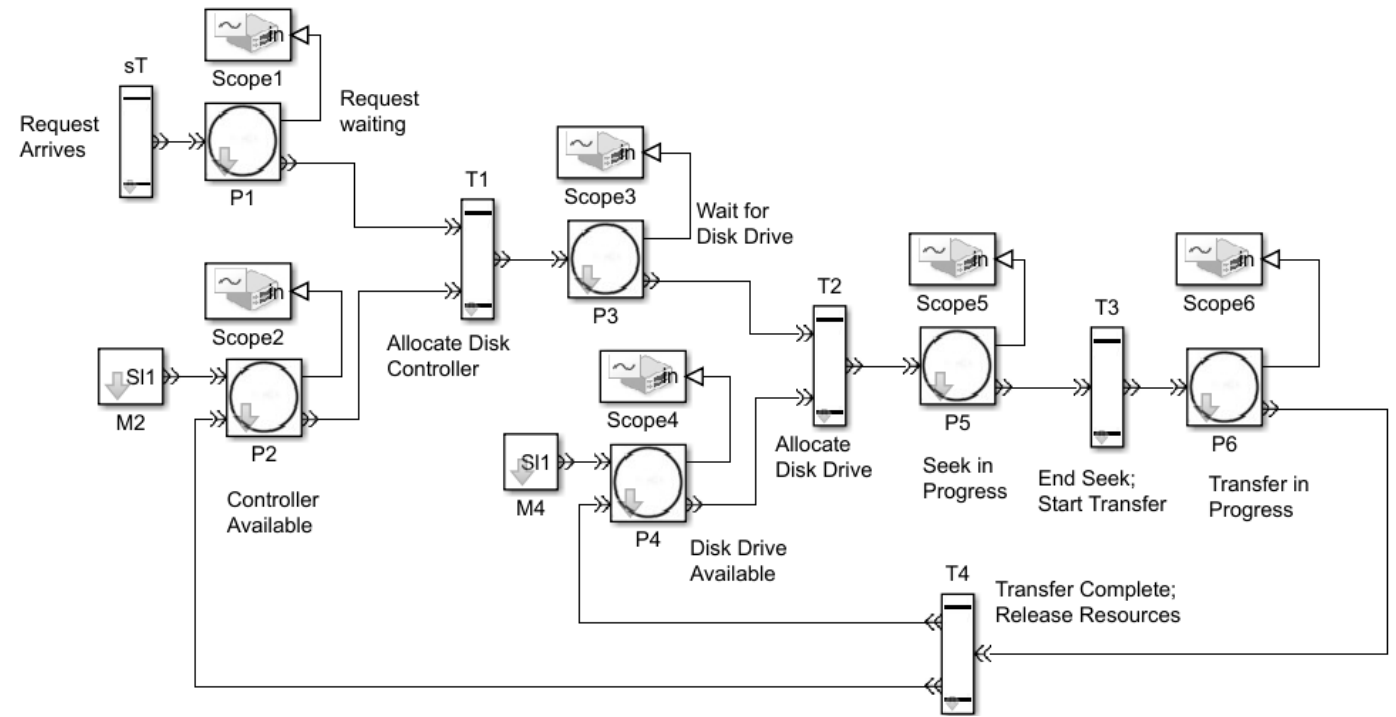

Figure 3: Blocks model representing a scheduling algorithm for allocation of disk controllers and drives.

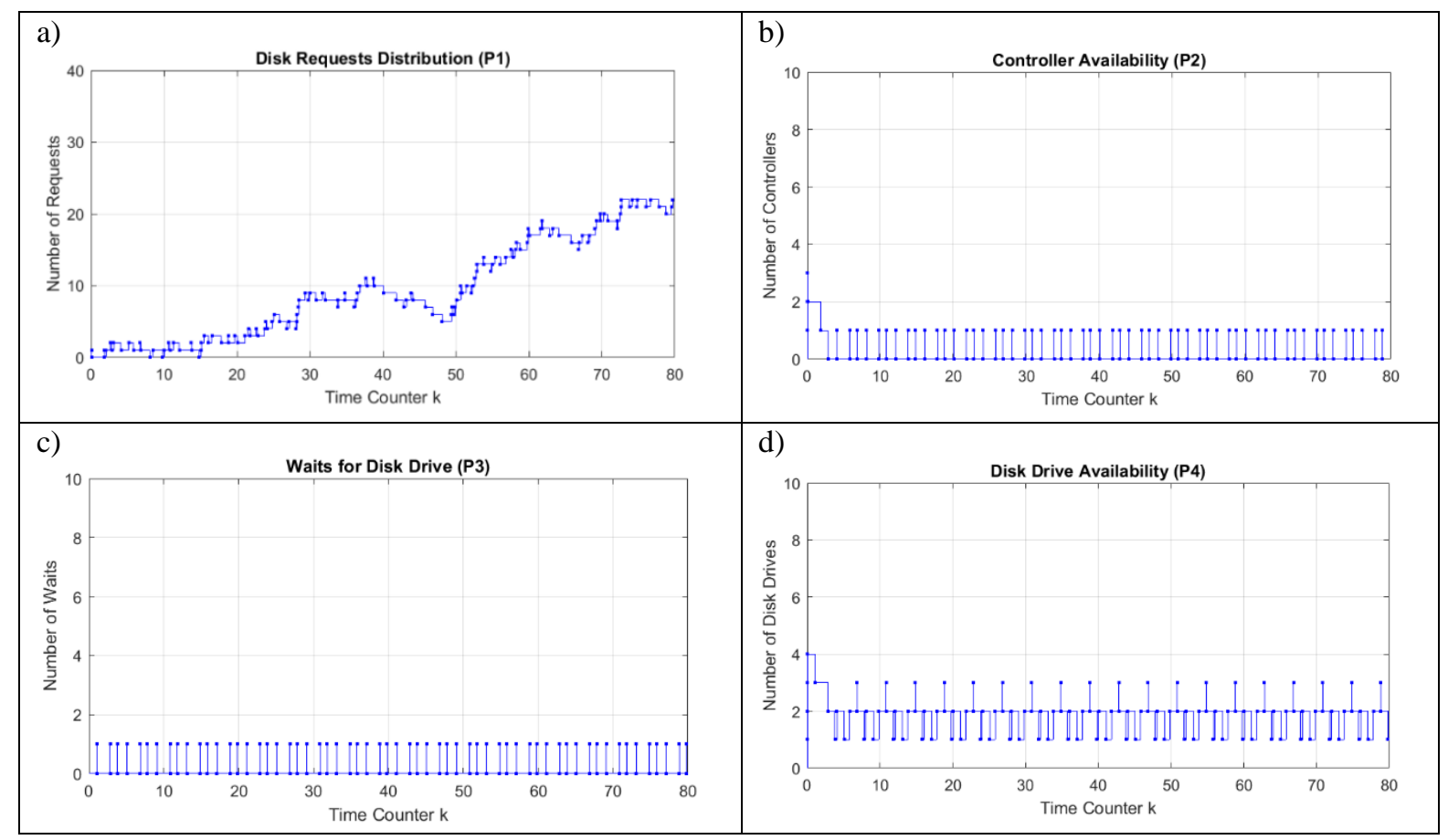

Figure 4: The plots of the simulation of the Petri net model representing the scheduling algorithm for allocation of disk controllers and drives with an exponential distribution in the disk request (sT).

Sink and Source Transition Block. The proposed implementation considers other two special types of transitions. The first additional block is a sink transition. This block is similar to a general transition, but instead of a replicate block, there is an entity sink. The inclusion of a sink transition helps for representing connections from the model to other non-included parts of the system that may be not relevant for the simulation process. Such transitions also help for preventing that the number of tokens in a place grows indefinitely, which could block 
a simulation process by the lack of computational resources. The main components of the sink transition block are depicted in Fig. 2 d. The last additional block considered in this work is a source transition. As in the case of a sink transition, the source transition allows for representing connections from other parts of the system that are irrelevant for a particular simulation process. The source transition subsystem is similar to the generic transition subsystem, but instead of having the combiner block there is an entity generator. Additionally, the user can configure the firing of a source transition to follow several probability distributions or even sophisticated algorithms implemented by Simulink blocks or functions. The main components of the source transition block are depicted in Fig. 2 e.

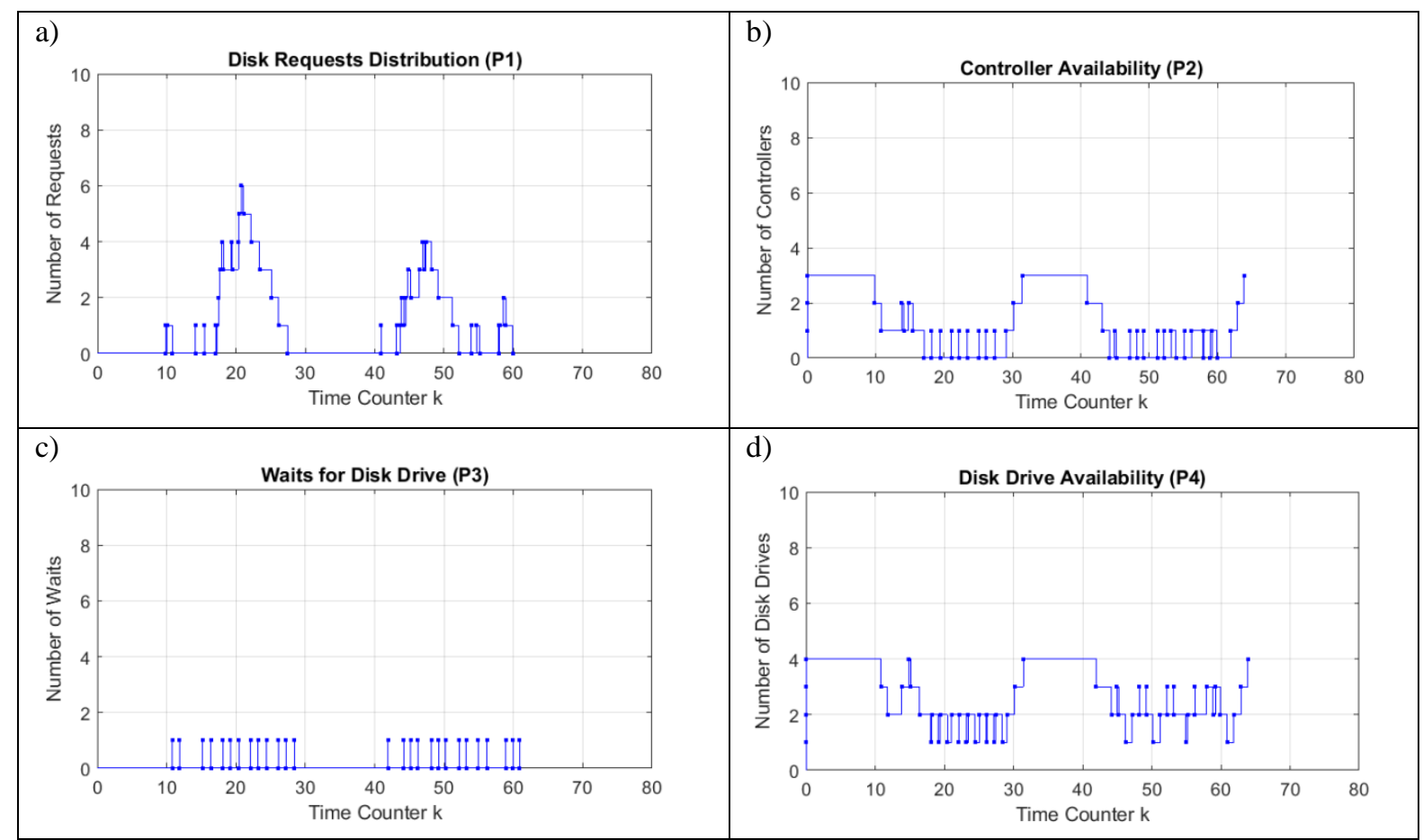

Figure 5: The plots of the Petri net model for the scheduling algorithm with a Log-logistic distribution in the disk request.

\section{APPLICATION EXAMPLES}

This section illustrates the application of the building blocks developed in the previous section for the construction of PN models reported in the literature. The aim is to show the potential use and the flexibility of the framework for the simulation of DES.

\subsection{Disk scheduling algorithm}

The disk-scheduling algorithm represented by the PN in Fig. 1 is built by the proposed blocks as shown in Fig. 3. It considers a number of disk drives and a number of disk controllers. This is captured by initial conditions $M 2$ and $M 4$ for places $P 2$ and $P 4$, respectively. The disk request arrives by the firing of transition $S T$. Notice that this transition has no input places, and then $S T$ transition can be tuned by selecting one of the probability distributions available for its firing, i.e., Poisson, Exponential, Bernoulli, Uniform, and Binomial, among others. The firing of $T 1$ means that the disk controller has been successfully allocated. Then, the system waits for a disk drive to be available. The firing of $T 2$ means that the disk drive has been successfully allocated and the seek operation is in progress. The firing of $T 3$ means that the 
seek operation is done and the system starts the transfer operation. Finally, the firing of $T 4$ means that the transfer operation is complete and the system is releasing the resources.

Fig. 4 shows the simulation results of this model for eighty clock cycles with an exponential distribution in the firing of $s T$. This distribution of the disk request is set through the parameters in the Matlab framework. The effect of this distribution is depicted in $P 1$ plot shown in Fig. 4 a. $P 3$ plot in Fig. $4 \mathrm{c}$ represents the waits of the request for the availability of disk drives. $P 2$ plot in Fig. $4 \mathrm{~b}$ and $P 4$ plot in Fig. $4 \mathrm{~d}$ represent the availability of disk controllers and disk drives, respectively. Observe that the availability of the controller starts at three, then goes down until zero, oscillates between one and zero, and finally goes back up to three, repeating the pattern. Similarly, the disk drive availability starts at four, then goes down until one, oscillates between one and two, and finally goes back up to four.

Fig. 5 shows the simulation results for a Log-logistic distribution in the firing of $s T$. The spikes in $P 1$ plot correspond with the decrease in the number of disk controllers and disk drives of $P 2$ and $P 4$ plots. The waits for disk drives $(P 3)$ follow a pattern that correspond with the distribution of the disk requests $(P 1)$. Every transition is set up with a service time of one time unit, for a pure event-driven simulation.

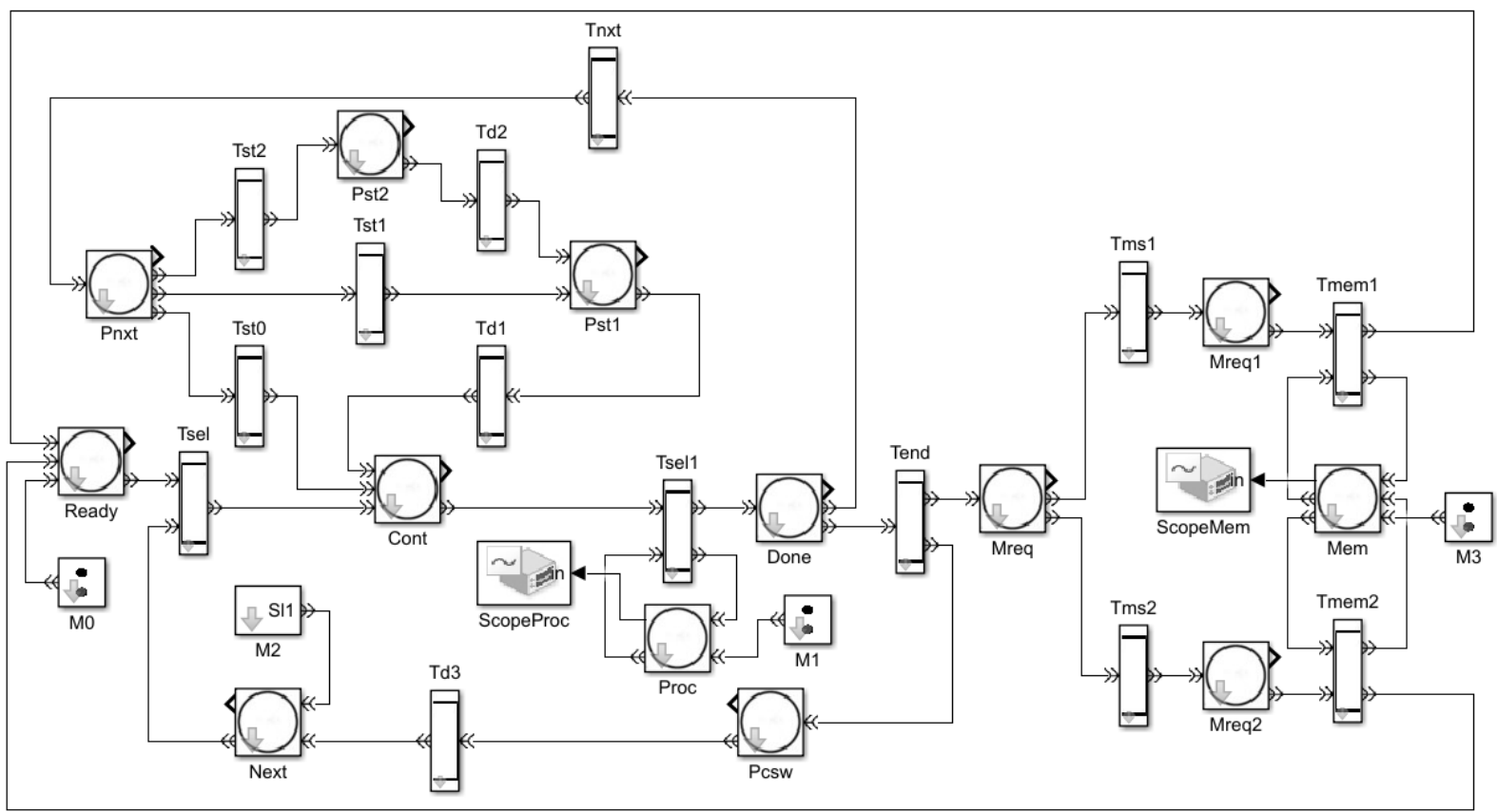

Figure 6: A Petri net model representing a multithreading architecture using the proposed blocks.

\subsection{Simultaneous multithreading architecture}

Fig. 6 depicts a Petri net as blocks model for a simultaneous multithreading architecture presented in [33]. Place Ready represents a pool of available threads. The firing of transition Tsel selects one of the ready threads, and then starts the first stage of the pipeline represented by one token on place Cont. Transition Trun denotes the execution of instructions or microinstructions. Place Proc connected to Trun transition characterises the available processing units. Thus, monitoring Proc place gives a measure of the CPU utilization. Done place allows the firing of Tend or Tnxt, if a given instruction performs a long-latency memory access or not, respectively. If an instruction does not perform a long-latency operation, then place Pnxt is marked which gives three options: continuing with the pipeline operation without no delay by firing transition Tst0; to perform a singly-cycle stall of the pipeline by firing transition Tst 1 ; or to perform a two-cycle stall of the pipeline by firing transition Tst2. If the instruction performs a long-latency operation, then places Pcsw and 
Mreq are marked. By one hand, the firing of transition Tcsw performs a context switch, and then a new thread could be selected by the marking of place Next. On the other hand, a token in place Mreq represents a memory request, which may derive in memory miss of level 1 by firing transition $T m s 1$, or in a memory miss of level 2 by firing transition Tms 2 . The marking of Mem place controls the number of ports for memory operation. Finally, the marking of place Next defines the number of simultaneous threads supported by the architecture. Noticeably, the markings of places Proc and Next are close related to the overall performance of the architecture. That is, two tokens on Next place have almost no impact on the performance of the architecture in the number of processing units, i.e. the marking of place Proc is not increased.

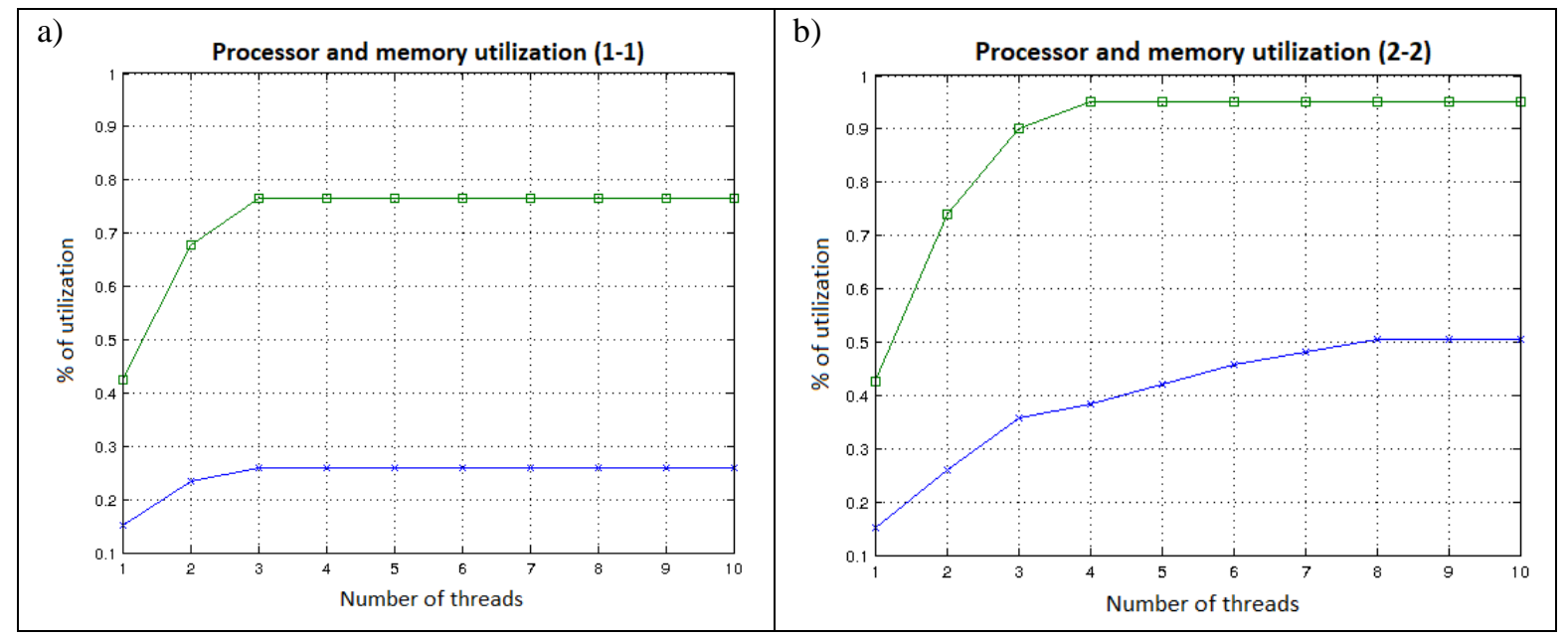

Figure 7: Multithreading architecture performance for the utilization of processor $(\boldsymbol{\square})$ and memory $(\times)$ : a) 1 unit -1 thread, b) 2 units -2 threads.

Fig. 7 shows the simulation results of two experiments with the model in Fig. 6. The first configuration considers one processing unit and one simultaneous thread, i.e. one token on place Proc and one token on place Next. The selection criteria for the output port of places Done, Pnxt and Mreq is configured with an equiprobable distribution and the default seed. The service time for all of the transitions is configured to one time unit. The number of threads is increased from one to ten. Fig.7 a depicts the performance curves for the utilization of processor $(\boldsymbol{\square})$ and memory $(x)$ for these parameters configuration. Processor utilization increases from above $40 \%$ with one thread to almost $80 \%$ with three threads. This performance remains the same from three threads up to ten threads, as depicted. Memory utilization slightly increases from $15 \%$ with one thread to almost $27 \%$ with three threads. Memory performance remains the same from three to ten threads.

Fig. $7 \mathrm{~b}$ shows results for a second configuration with two processing units and two simultaneous threads, i.e., two tokens in place Proc as well as two tokens in place Next. In this case, the processor utilization increases from below $45 \%$ with one thread up to $95 \%$ with four threads. From four to ten threads, the processor utilization remains constant at $95 \%$. The memory utilization rises from $15 \%$ with one thread to almost $37 \%$ with three threads. Then, it goes above $40 \%$ with five threads, and finally up to $50 \%$ of utilization with eight threads. From eight to ten threads, this value remains constant.

With an equiprobable distribution in the selection of the output ports of places Done, Pnxt and Mreq, the performance curves obtained from the simulations of the model in Fig. 9 correspond reasonably well with those reported in [33]. All of transitions were configured to a service time of one time unit, which may represent a clock tick in a real architecture. 


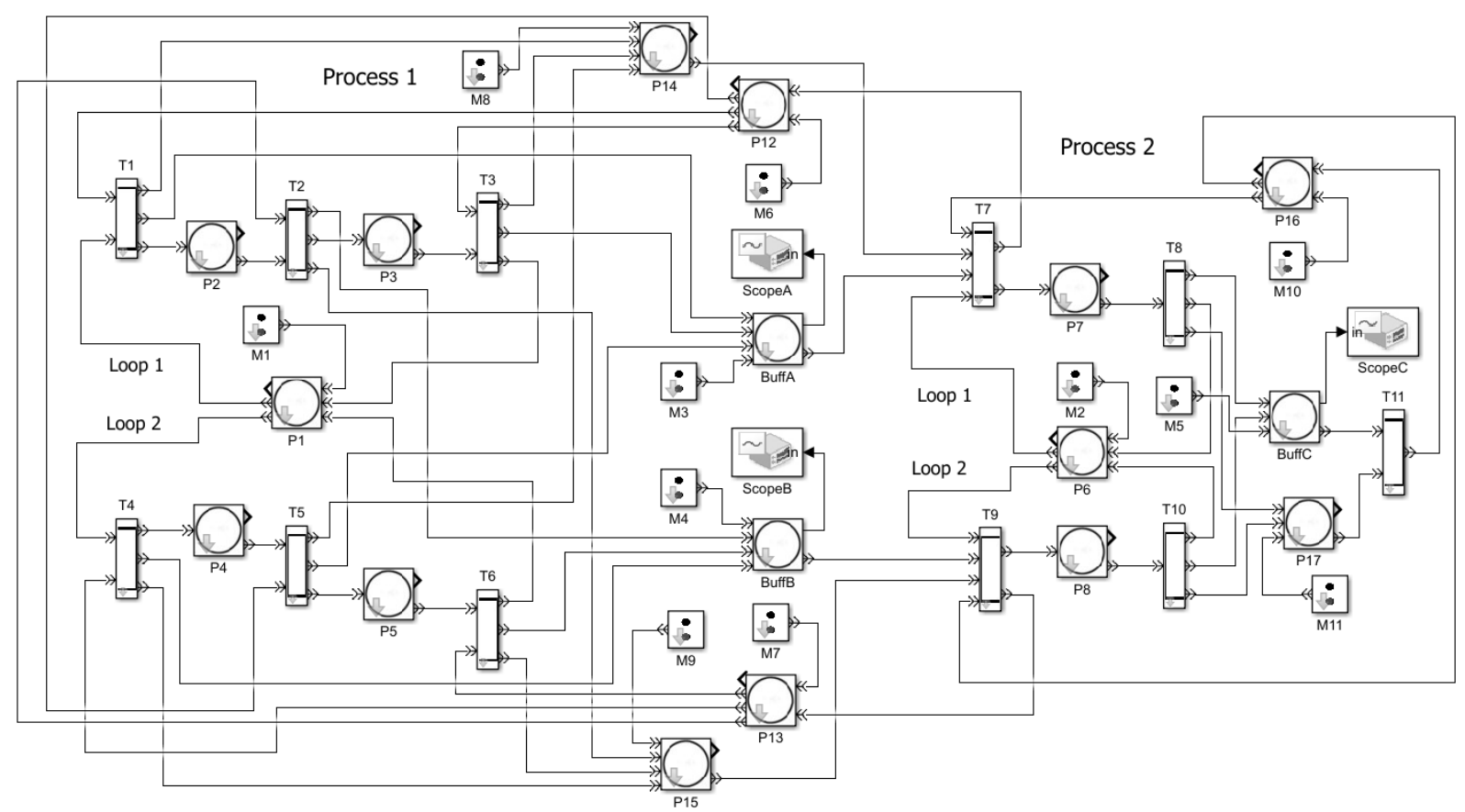

Figure 8: A Petri net model for the supervisory control of the buffer levels in communicating processes.

\subsection{Supervisory control of communicating processes}

The block model in Fig. 8 represents a communicating processes system. Authors reported this system as an application example modelled as PN in [34]. Process 1 and Process 2 mainly compose the system. Two execution loops, Loop 1 and 2, conform each of the processes. Loops in every process may be arbitrarily executed. Output ports of place P1 are configured with an equiprobable distribution for selecting the firing of either, T1 or T4, which corresponds to the execution of Loop 1 or 2, respectively. Similarly, for Process 2, an equiprobable distribution is used for the selection of the output port of place P6, which decides the execution of Loop 1 by firing T7 or Loop 2 by firing T9. Buffer C connects with Process 2 by transition T11. The transition T11 drains the tokens from buffer $\mathrm{C}$ and allows representing further connections with other subsystems in a more complex model.

Places P12, P13 and P14, referred as upper control places, allow for controlling the upper level of buffers A, B and C, respectively. Every buffer and its upper control place conform to one conservative component in the model, i.e, P12 and BuffA, P13 and BuffB, and P14 and BuffC, are the three conservative components for upper bounds. The sum of tokens at initial marking, of any buffer and its upper control place, defines the number of tokens on the component that will remain constant for any evolution of the controlled net, as stated in [34]. Similarly, places P15, P16 and P17, referred as lower control places, allow for controlling the lower level of buffers A, B and C, respectively. As shown, these places are almost reverse connected with respect to the places controlling the upper level of the buffers. Once more, every lower control place and its corresponding buffer place conform a conservative component, i.e, P15 and BuffA, P16 and BuffB, and P17 and BuffC, are the three conservative components for lower bounds. The difference at initial marking, between the number of tokens of the buffer and the number of tokens in its lower control place, defines the lower bound of the involved buffer. 


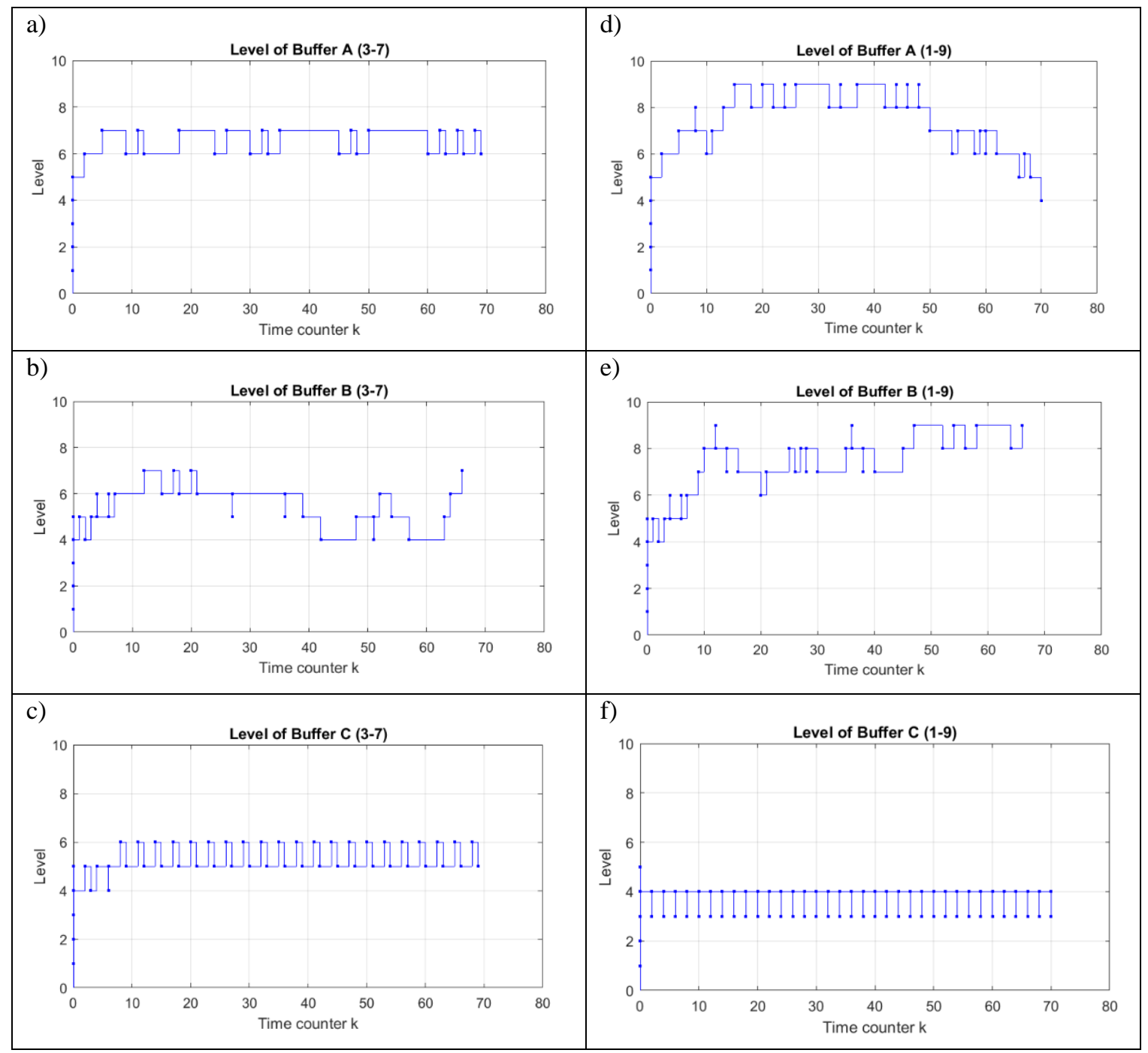

Figure 9: The simulation results for the communicating processes model.

Fig. 9 depicts some simulation results of the blocks model for the supervisory control of the buffer levels in communicating processes. Plots in Figs. 9 a to $9 \mathrm{c}$ show the results on levels of buffers A, B and C for one configuration. Plots in Figs. $9 \mathrm{~d}$ to $9 \mathrm{f}$ show the results for a second configuration of the model.

For the first configuration, the initial condition of all buffers is five tokens each, while the initial condition of control places is two each. These initial conditions correspond to desired bounds for buffers A, B and C, given a lower level of 3 and an upper level of 7. Every transition in the model is configured with a service time of one time unit, except for T11 with a service time of three time units. In addition, P1 and P6 are configured with an equiprobable distribution for the selection of output ports for allowing an arbitrary execution of loops in Process 1 and 2. Observe that buffer $\mathrm{C}$ in plot in Fig. $9 \mathrm{c}$ produces a quasi-constant high-level. Therefore, the firing of T11 may require bigger service time units than three.

For the second configuration, the initial conditions of all buffers remain the same, while the initial condition of control places is set to 4 each. Therefore, due to the conservative components for controlling the upper and lower bounds, it results on a lower level of 1 and an upper level of 9. Service time of T11 is set to two time units. P1 and P6 are configured with an equiprobable distribution just as previous configuration. Observe the level of buffer $\mathrm{C}$ in plot in Fig. $9 \mathrm{f}$, which shows the effect of the reduction of service time to two time units. 
Levels of buffers A and B are almost at their upper bound due to the accumulation of tokens produced by Process 1, which Process 2 is unable to consume at the same rate.

\section{CONCLUSION}

This paper proposes the development of a Matlab/Simulink framework for the simulation of PN models. Some SimEvents blocks were integrated into Simulink subsystems representing the basic elements of a PN. A generic PN place, transition, and initial marking were developed capturing the semantics of a PN. The construction of Petri net models is quite simple and intuitive by using the developed components. The ability for including a PN within a Simulink model, the visualization of the model as a PN indeed, and the wide variety of tools in Matlab that can be used for simulation of PN models, are some advantages of the proposed framework. Three application examples reported in the literature were developed and simulated within the proposed framework illustrating its flexibility and simplicity in the construction of models for DES. The framework offers the availability of construct the input and output matrices of the built PN through $m$-function programming. Therefore, multiple analyses based on the incidence matrix can be performed based on the model built with the proposed blocks, such as reachability set, place or transition invariants, and many others. The library is free for download at Matlab Central at:

https://www.mathworks.com/matlabcentral/fileexchange/50571-petri-nets-building-blockslibrary.

\section{REFERENCES}

[1] Reisig, W. (2013). Understanding Petri Nets: Modelling Techniques, Analysis Methods, Case Studies, Springer, Heidelberg

[2] Iordache, M.; Antsaklis, P. J. (2007). Supervisory Control of Concurrent Systems: A Petri Net Structural Approach, Springer, Basel

[3] Hrúz, B.; Zhou, M. (2007). Modelling and Control of Discrete-Event Dynamic Systems: With Petri Nets and Other Tools, Springer, London

[4] Desel, J.; Esparza, J. (2005). Free Choice Petri Nets, Cambridge University press, Cambridge

[5] Vučak, I.; Car, Ž.; Vuković, M. (2017). User interface adaptation for ICT based alternative and augmentative applications, Technical Gazette, Vol. 24, No. 1, 29-38, doi:10.17559/TV20140623205344

[6] David, R.; Alla, H. (2005). Discrete, Continuous, and Hybrid Petri Nets, Springer, Berlin

[7] Jensen, K. (2013). Coloured Petri Nets: Basic Concepts, Analysis Methods and Practical Use, $2^{\text {nd }}$ edition, Springer, Berlin

[8] Jensen, K.; Rozenberg, G. (2012). High-level Petri Nets: Theory and Application, SpringerVerlag, Berlin

[9] Haas, P. J. (2006). Stochastic Petri Nets: Modelling, Stability, Simulation, Springer Science \& Business Media, New York

[10] Liu, Z.; Liu, Y.; Cai, B.; Li, J.; Tian, X. (2017). Reliability analysis of multiplex control system of subsea blowout preventer based on stochastic Petri net, Technical Gazette, Vol. 24, No. 1, 714, doi:10.17559/TV-20130502140334

[11] Agha, G. A.; De Cindio, F.; Rozenberg, G. (2001). Concurrent Object-Oriented Programming and Petri Nets: Advances in Petri Nets, Springer-Verlag, Berlin

[12] Li, Z.; Zhou, M. (2009). Deadlock Resolution in Automated Manufacturing Systems: A Novel Petri Net Approach, Springer-Verlag, London

[13] Reisig, W. (2013). Elements of Distributed Algorithms: Modelling and Analysis with Petri Nets, Springer-Verlag, Berlin

[14] Zaitsev, D. (2013). Clans of Petri Nets: Verification of Protocols and Performance Evaluation of Networks, Lap Lambert Academic Publishing, Saarbrücken 
[15] Van der Aalst, W.; Desel, J.; Oberweis, A. (2003). Business Process Management: Models, Techniques, and Empirical Studies, Springer-Verlag, Berlin

[16] Xiao, N.; Ni, C. D.; Guo, S. J. (2017). Modelling and simulation for production logistics system in industrial enterprises based on hybrid network, International Journal of Simulation Modelling, Vol. 16, No. 1, 157-166, doi:10.2507/IJSIMM16(1)CO3

[17] Kavicka, A.; Klima, V.; Adamko, N. (2007). Simulations of transportation logistics systems utilising agent-based architecture, International Journal of Simulation Modelling, Vol. 6, No. 1, 13-24, doi:10.2507/IJSIMM06(1)2.075

[18] Yakovlev, A.; Gomes, L.; Lavagno, L. (2000). Hardware Design and Petri Nets, Springer Science \& Business Media, Dordrecht

[19] Petri Nets World. Guth's Petri Net Java Applet, from http://www.informatik.unihamburg.de/TGI/PetriNets/tools/java/Guth/, accessed on 23-05-2018

[20] Petri Nets World. Bräunl's Petri Net Java Applet, from http://www.informatik.unihamburg.de/TGI/PetriNets/tools/java/Braunl/, accessed on 23-05-2018

[21] Petri Nets World. Patrice's Petri Net Java Applet, from http://www.informatik.unihamburg.de/TGI/PetriNets/tools/java/Patrice/, accessed on 23-05-2018

[22] Laboratoire d'Informatique de Paris 6. CPN-AMI, from http://move.lip6.fr/software/CPNAMI/, accessed on 23-05-2018

[23] Eindhoven University of Technology. CPN Tools, from http://www.daimi.au.dk/CPNTools/, accessed on 23-05-2018

[24] Dipartimento di Informatica e Automazione. GDToolkit, from http://www.dia.uniroma3.it/ gdt/gdt4/index.php, accessed on 23-05-2018

[25] Università di Torino. GreatSPN, from http://www.di.unito.it/ greatspn/, accessed on 23-05-2018

[26] Humboldt-Universität zu Berlin. INA: Integrated Net Analyzer, from http://www2.informatik.huberlin.de/ starke/ina.html, accessed on 23-05-2018

[27] Helsinki University of Technology. Maria: The Modular Reachability Analyser, from http://www.tcs.hut.fi/Software/maria/, accessed on 23-05-2018

[28] Rwth Aachen University. Petrinet-tool Netlab, from http://www.irt.rwthaachen.de/cms/IRT/Studium/Downloads/ osru/Petrinetz-Tool-Netlab/?lidx=1, accessed on 2305-2018

[29] Asachi Technical University of Iasi. Petri Net Toolbox, from http://www.mathworks.com/products/connections/product_detail/product_35741.html, accessed on 23-05-2018

[30] University of Zaragoza. SimHPN, from https://www.informatik.unihamburg.de/TGI/PetriNets/tools/db/simhpn.html, accessed on 23-05-2018

[31] Ralston, A.; Reilly, E. D. (1993). Encyclopaedia of Computer Science, $3^{\text {rd }}$ edition, Van Nostrand Reinhold Co., New York

[32] Mathworks. SimEvents: User's Guide (R2018b), from https://la.mathworks.com/help/pdf_doc/simevents/simevents_ug.pdf, accessed on 23-01-2018

[33] Zuberek, W. M. (2012). Timed Petri nets in performance exploration of simultaneous multithreading, Pawlewski, P. (Ed.), Petri Nets-Manufacturing and Computer Science, IntechOpen, London, 299-312, doi: 10.5772/48601

[34] Campos-Rodríguez, R.; Alcaraz-Mejia, M.; Mireles-Garcia, J. (2007). Supervisory control of discrete event systems using observers, Proceedings of the 2007 Mediterranean Conference on Control \& Automation, 1-7, doi:10.1109/MED.2007.4433816 